\title{
Comparison of spinal cord evoked potentials and peripheral nerve evoked potentials by electric stimulation of the spinal cord under acute spinal cord compression in cats
}

\author{
M Arai*,1, ${ }^{\text {T Goto }}{ }^{1}$, A Seichi ${ }^{1}$, T Miura $^{1}$ and K Nakamura ${ }^{1}$ \\ ${ }^{1}$ Department of Orthopaedic Surgery, Faculty of Medicine, The University of Tokyo, Tokyo, Japan
}

\begin{abstract}
Study design: Spinal cord evoked potentials and peripheral nerve evoked potentials after spinal cord stimulation were recorded under acute spinal cord compression in 19 cats.

Objectives: To investigate the effects of acute compression upon grey matter and white matter by comparing both potentials.

Methods: We compared peripheral nerve evoked potentials, recorded at the biceps brachii branch of the musculocutaneous nerve, with descending spinal cord evoked potentials, recorded from the lumbar spinal cord, by stimulation to the $\mathrm{C} 2$ level, under compression of the C6 segment.

Results: The amplitude of both potentials decreased with increased compression. The second wave of peripheral nerve evoked potentials, which are motor fibre action potentials, decreased sooner than those of the spinal cord evoked potentials.

Conclusion: These findings indicate that peripheral nerve evoked potentials are sensitive to acute damage of the segmented compression. This suggests that grey matter is more vulnerable to compression than white matter.
\end{abstract}

Spinal Cord (2000) 38, $403-408$

Keywords: spinal cord evoked potentials; peripheral nerve evoked potentials; acute spinal cord compression; biceps brachii nerve; grey matter; white matter

\section{Introduction}

For intraoperative monitoring of spinal cord evoked potentials, conventional methods, such as spinal cord evoked potentials by stimulation of the spinal cord (SpSCEP), or somatosensory evoked potentials, do not detect motor tract function. Motor evoked potentials (MEP) are more appropriate for this purpose. Since the motor tract conducts through synapses at the anterior horn of the grey matter, evoked potentials recorded in peripheral nerves, or muscles, represent motor pathways, including grey matter. In spite of many papers about spinal cord evoked potentials in a spinal compression model, there have been no reports investigating the function of the grey matter under direct compression electrophysiologically. We observed the change of action potentials in peripheral nerves elicited by compression of the spinal cord segment, which contains most of the neurons of peripheral nerves. In cats, cell somata of the biceps brachii muscle branch of musculocutaneous nerve are mainly located in the C6 segment of the spinal cord. ${ }^{1,2}$

*Correspondence: M Arai, Department of Orthopaedic Surgery, Faculty of Medicine, The University of Tokyo, 7-3-1 Hongo, Bunkyo-ku, Tokyo, 113-0033, Japan
Therefore, action potentials recorded in the biceps brachii muscle branch under acute compression of the C6 segment reflect directly the function of grey matter under acute compression. We stimulated the upper cervical cord at the $\mathrm{C} 2$ level and recorded spinal cord evoked potentials at the lumbar spinal cord, which represents the descending conductive spinal cord potentials (Sp-SCEP), and at the biceps brachii branch of the musculocutaneous nerve, which represents the peripheral nerve evoked potentials (SpPnEP). The purpose of this study is to investigate electrophysiological effects upon the grey matter and white matter by comparing Sp-SCEP with Sp-PnEP, when the spinal cord is compressed in cats.

\section{Materials and methods}

Animal preparations

Nineteen cats, weighing $2.3-4.5 \mathrm{~kg}$, were used for the experiments. Animals' care conformed to the principles approved by the Guide for Animal Experimentation, Faculty of Medicine, the University of Tokyo. Atropine sulphate $(0.5 \mathrm{mg} /$ body $)$ and ketamine $(50 \mathrm{mg} / \mathrm{kg})$ were administered intramuscularly. A tracheostomy was performed and the animals were 
anaesthetized with halothane vaporized in a mixture of oxygen and nitrogen. The left femoral vein was catheterized for infusion of fluid and administration of required drugs. The animals were immobilized by intravenous administration of pancuronium bromide $(0.1 \mathrm{mg} / \mathrm{kg} / \mathrm{h})$ and artificially ventilated. The blood pressure was monitored through a catheter inserted into the left femoral artery and maintained above $100 \mathrm{mmHg}$, with a small amount of Neo-synesin ${ }^{(\mathrm{R})}$, when necessary. The brachial plexus was exposed and the motor branch of the musculocutaneous nerve to the biceps brachii muscle was identified and dissected free. The animals were fixed in a stereotaxic frame in the sphinx position. The vertebral column was immobilized using a vertebral body clamp at L3 level. Laminectomies were performed at the cervical $(\mathrm{C} 2-\mathrm{C} 7)$ and lumbar (L2) vertebral levels. The dura matar was incised to expose the spinal cord. Body temperature was monitored with a rectal thermometer and maintained at the range of $36.0-37.0^{\circ} \mathrm{C}$, using a heating blanket and infrared lamp, as required.

\section{Stimulation procedure}

A tungsten electrode $(300 \mu \mathrm{m}$ in diameter $)$ was positioned along the mid-line at a depth of $1 \mathrm{~mm}$ at the $\mathrm{C} 2$ level. Stimuli were repetitive $(3 \mathrm{~Hz})$ rectangular wave pulses of $300 \mu \mathrm{sec}$ duration, $0.8-2 \mathrm{~mA}$ intensity. (Electronic Stimulator: SEN-7203, Isolator: SS-701J, Nihon Kohden Co., Japan)

\section{Recording procedure}

Sp-SCEP were recorded using a tungsten electrode (300 $\mu \mathrm{m}$ in diameter) positioned at the mid-line at $1 \mathrm{~mm}$ at the L2 level. Impedance of the recording electrode was kept below $100 \mathrm{k} \Omega$. Sp-PnEP were recorded with a bipolar silver wire electrode at the dissected biceps motor branch of the musculocutaneous nerve to record peripheral nerve evoked potentials. Repetitive recorded 128 evoked potentials were added and averaged, then displayed on an amplified oscilloscope (VC-11 Nikon Kohden Co., Japan) and recorded using a computer (Apple Macintosh, Maclab8). Cut-off filters were set at $15 \mathrm{~Hz}-3 \mathrm{kHz}$. The earth lead was attached to nearby paravertebral muscles at each stimulation and recording level.

\section{Root lesioning method}

In one cat we incised the dorsal roots of $\mathrm{C} 6, \mathrm{C} 5$ and $\mathrm{C} 7$ followed by the incision of the ventral roots of $\mathrm{C} 6$, C5 and C7, under microscopy, to observe the changes of Sp-PnEP of the biceps brachii motor branch.

\section{Compression experiment}

The C6 spinal segment was identified to position the compression device. The device was made of acrylic rod with a square $(5 \times 5 \mathrm{~mm})$ smooth surface attached to the spinal cord. Spinal cord compression at the C6 segment was performed, using this rod equipped with a micromanipulator. Compression was gradually increased by $0.5 \mathrm{~mm}$ per $3 \mathrm{~min}$ to $3 \mathrm{~mm}$, while monitoring Sp-SCEP and Sp-PnEP at each step (Figure 1). The first and second waves of both $\mathrm{Sp}$ SCEP and Sp-PnEP were measured as follows: the amplitude was the height of the positive wave from the baseline, and the latency was the time to the positive peak of each wave from the onset of stimulus (Figure 2). The changes of amplitude and latency under compression were compared with those of the original recorded waves before compression.

All results were presented as means \pm SD. Statistical analysis of the results were evaluated using analysis of unpaired $t$-test (Student's $t$-test) followed by unpaired $t$-test to evaluate changes among individual groups. A $P$-value less than 0.05 was considered statistically significant.

\section{Histological procedure}

The animals were euthanized with an overdose of pentobarbital sodium, administered intravenously after the experiments were finished. After postfixation with $10 \%$ formalin, their cervical spinal cords, between $\mathrm{C} 2$

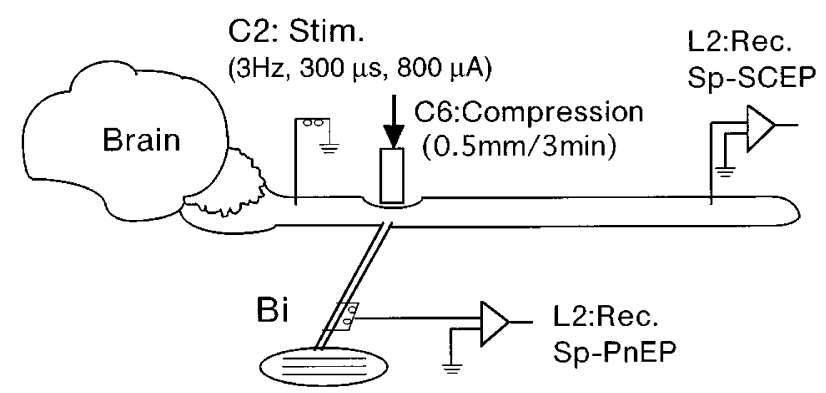

Figure 1 The scheme of our experiment. Sp-SCEP is elicited by $\mathrm{C} 2$ stimulation and recorded at L2 spinal cord. Sp-PnEP is elicited by $\mathrm{C} 2$ stimulation and recorded at the biceps brachii muscle branch of the musculocutaneous nerve, C6 segment is compressed gradually where biceps motoneurone pool exists

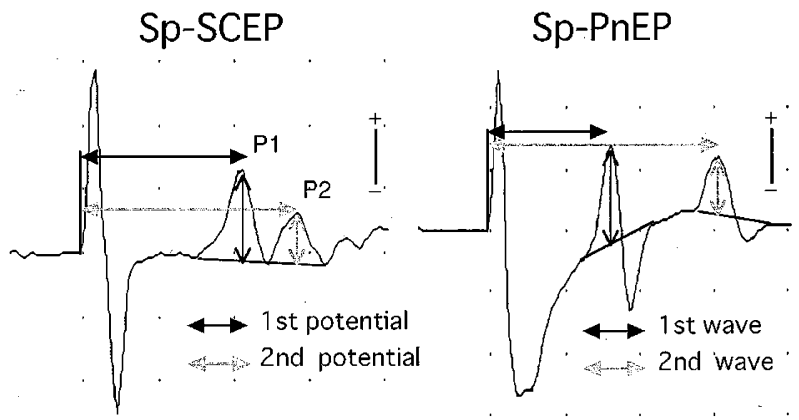

Figure 2 The original wave form of Sp-SCEP and Sp-PnEP. Both potentials consist of two waves, each amplitude is measured as wave height from the baseline, and latency as time to peak from the onset of stimulus 
and $\mathrm{C} 7$, were resected en bloc. Transverse serial sections of the spinal cord $(100 \mu \mathrm{m}$ thick) were made and stained with luxor fast blue and cresyl violet (Klüver-Barrera stain).

\section{Results}

Change of $S p-P n E P$ with root resection

$\mathrm{Sp}-\mathrm{PnEP}$ was composed of two waves. The first wave had $1.6 \mathrm{msec}$ of latency, and the second wave had $3.1 \mathrm{msec}$ of latency. By high frequency stimulation at $40 \mathrm{~Hz}$, the second wave disappeared, whilst the first wave followed to stimulation. The amplitude of the first wave decreased to $50 \%$ by $\mathrm{C} 6$ dorsal root resection, was unchanged by $\mathrm{C} 5$ dorsal root resection and disappeared by C7 dorsal root resection. The amplitude of the second wave decreased to $37.5 \%$ by C6 ventral root resection, was unchanged by $\mathrm{C} 5$ ventral root resection and disappeared by $\mathrm{C} 7$ ventral root resection. The latency of both waves did not change. The origin of Sp-PnEP is considered as follows: the first wave is the action potential of group Ia fibres which run through the C6 dorsal root and partially ascend in the posterior funiculus. The second wave is the action potential of motoneurons in the anterior horn of the C6 segment, which reflects the function of the grey matter, especially of the intermediate layer and anterior horn of the C6 segment (Figure 3).

\section{Change of Sp-SCEP and Sp-PnEP with spinal cord} compression

Figure 4B shows the average change of four waves amplitude with compression. Sp-SCEP represents the sensory tract via white matter. The amplitude and latency of the first potential were analyzed because the first potential is more stable and its amplitude is larger than that of the second potential. Therefore, we compared the first positive potential with the second wave of Sp-PnEP. The amplitude of the first potential slightly increased to $104.7 \%$ with less than $1 \mathrm{~mm}$ compression and decreased to $78.0 \%$ at $3 \mathrm{~mm}$ compression. Otherwise the amplitude of the second wave of Sp-PnEP decreased gradually from the early step to $64.8 \%$ at $3 \mathrm{~mm}$ compression. Between the two groups there was a significant difference $(P<0.05)$ at each compression step, except for $3 \mathrm{~mm}$. The delay of latency in both groups was subtle and there was no significant difference (Figure 5).

Figure 4A shows the example case of actual potential wave change.

\section{Histological findings}

There were no abnormal findings such as haemorrhage, necrosis in the grey matter or demyelination in the white matter in sectional slices of the compressed site (Figure 6).

\section{Discussion}

There are various methods for intraoperative monitoring. Overall, conventional methods are used to monitor spinal cord tracts. For example, conductive spinal cord evoked potentials by stimulation of the spinal cord ( $\mathrm{Sp}$ SCEP), which pass through white matter tracts, are conventional in Japan. Somatosensory evoked potentials (SEP), which also pass through the ascending sensory tract, are commonly used in America and Europe. To monitor the motor tract, especially the pyramidal tract, motor evoked potentials (MEP) have recently been developed. There are various methods defined by stimulation and recording sites along the motor tract pathway. The motor tract arises from the motor cortex, or brain stem nucleus, descends down through the white matter, then transmits synapses at the anterior horn of the grey matter, so the motoneuron sends axon to the target muscles via a neuromuscular juction. Each MEP represents a part of the motor tract, but none of them is sufficient to monitor grey matter function entirely. For this purpose $F$ wave is a motoneuron firing by antidromically ascending impulses along the alpha-fibre, and $\mathrm{H}$ reflex

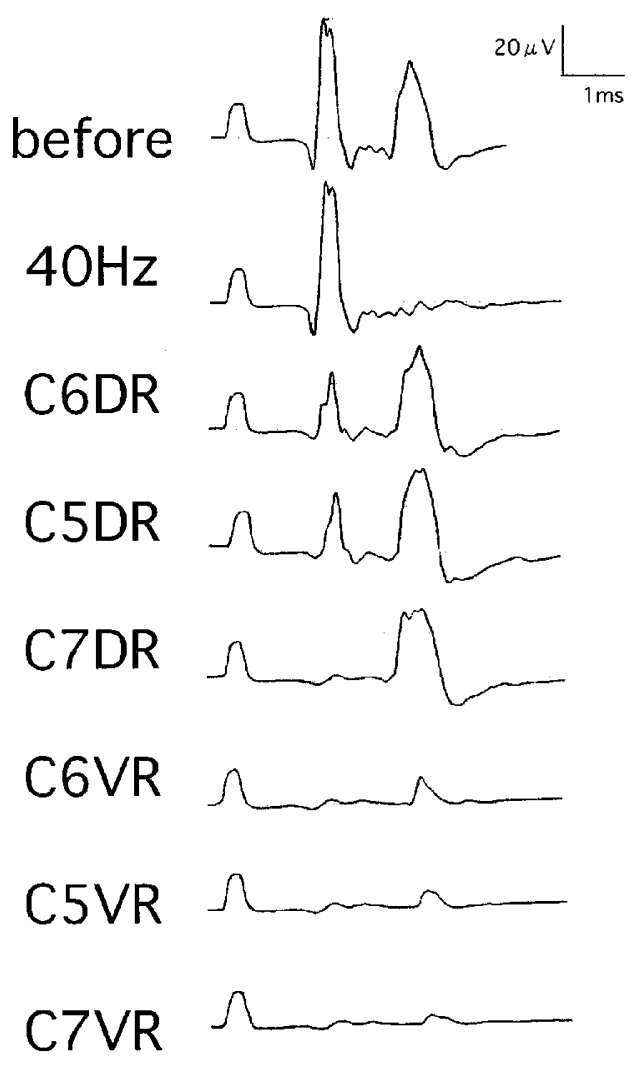

Figure 3 The change of Sp-PnEP by resection of nerve roots. The high frequency stimulation $(40 \mathrm{~Hz})$ made the second wave disappear but the first wave followed to the stimulation. The amplitude of the first wave decreased to $50 \%$ by $\mathrm{C} 6$ dorsal root resection, and that of the second wave decreased to $37.5 \%$ by $\mathrm{C} 6$ ventral root resection 
reflects motoneuron activity excited by impulses conducted along group Ia fibre. They both detect the reflex pathway and do not reflect the grey matter function directly.

On the other hand, according to the Bell-Magendie law, the ventral root consists chiefly of motor axons, including alpha-motor axons, gamma-motor axons, and at certain segmental levels, autonomic preganglio-

a

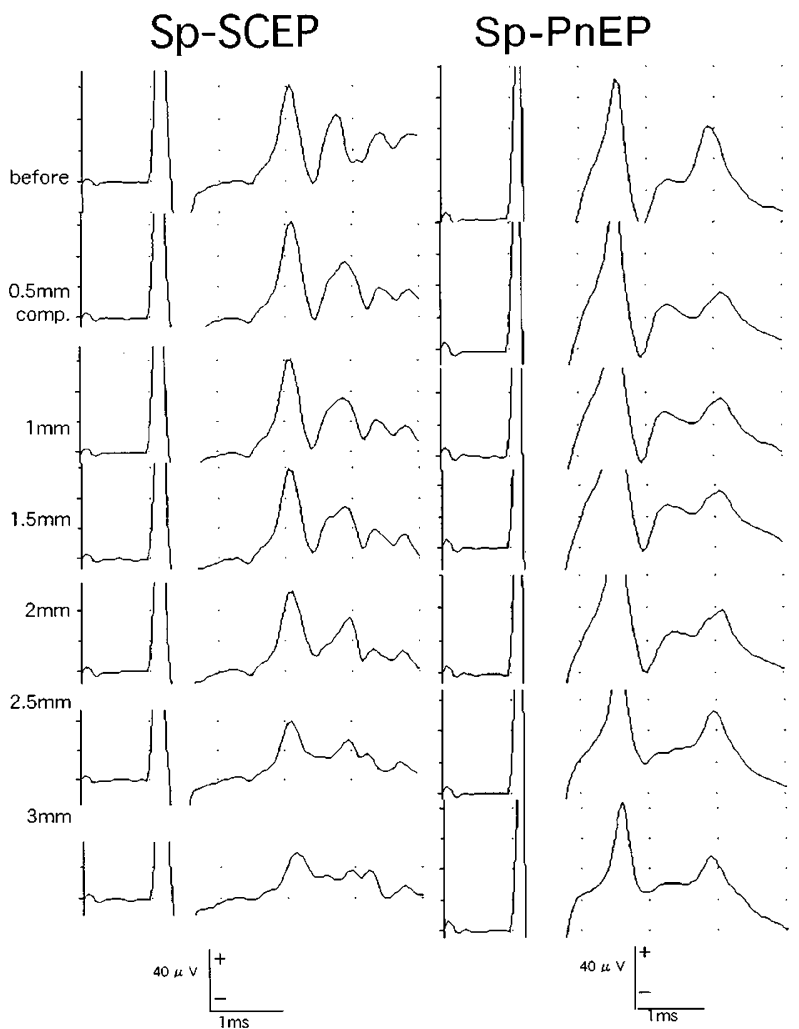

b

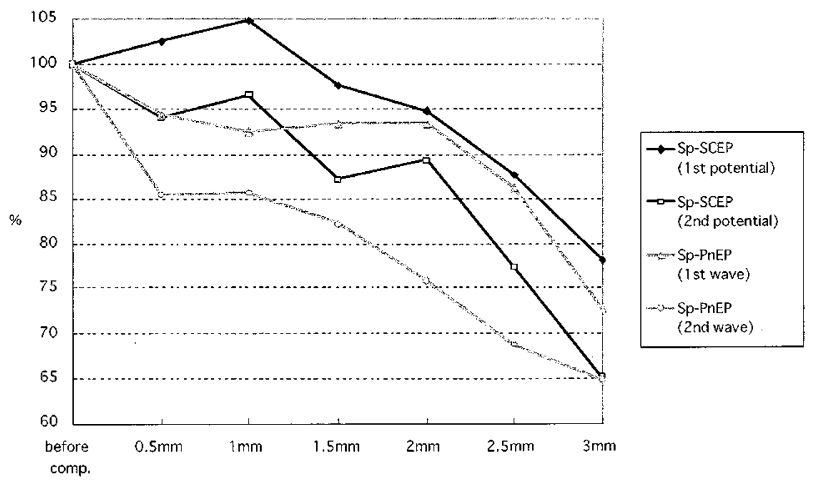

Figure 4 (a) The change of both waves under compression. Example case. (b) The change of averaged amplitude under compression. The amplitude of the first potential of Sp-SCEP slightly increased to $104.7 \%$ with less than $1 \mathrm{~mm}$ compression and decreased to $78 \%$ at $3 \mathrm{~mm}$ compression. Other waves decreased gradually; the second potential to $65.1 \%$, the first wave of Sp-PnEP to $72.7 \%$, the second wave to $64.8 \%$ at $3 \mathrm{~mm}$ compression
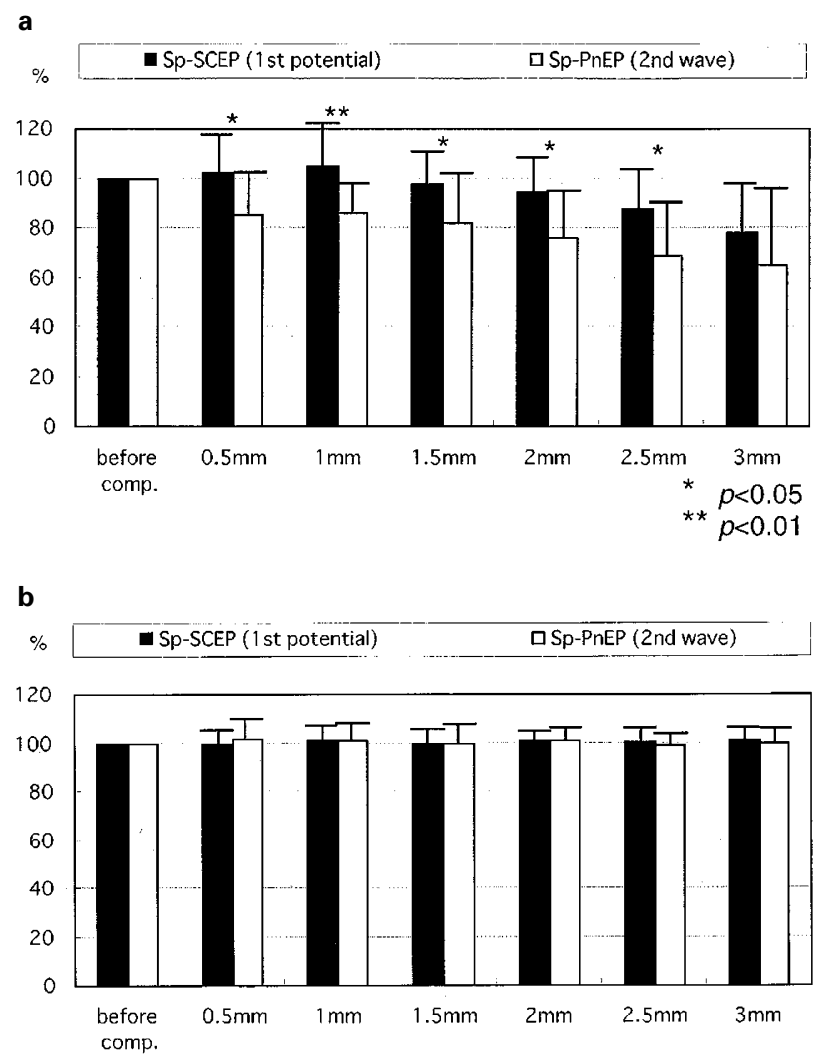

Figure 5 The comparison between the first potential of SpSCEP and the second wave of Sp-PnEP. (a) The change of amplitude under compression. There were significant differences $(P<0.05)$ at each compression step except for $3 \mathrm{~mm}$. (b) The change of latency under compression. The delay of latency of both groups were subtle and there were no significant differences

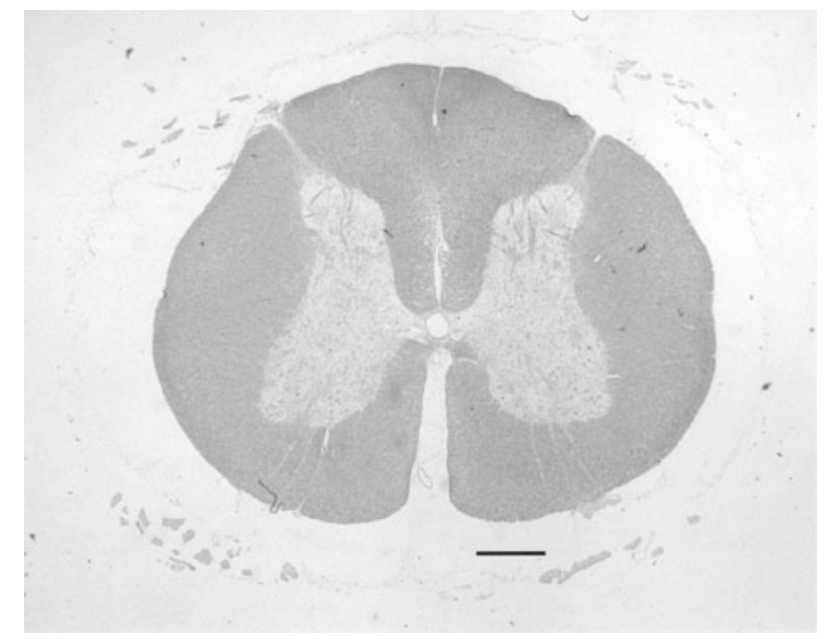

Figure 6 Histological findings in the compressed site (C6 segment). There were normal findings without grey matter haemorrhage or axonal demyelination. Calibration bar indicates $1 \mathrm{~mm}$ 
nic axons. Ventral roots also contain a few primary afferent fibres, whose role is still unclear. But these afferent fibres and autonomic preganglionic fibres are mainly demyelinated axons, classified as group IV, or type $\mathrm{C}$ fibres. The velocity of these fibres is much slower than that of motor fibres. In our experiment the velocity of the second wave is approximately $20 \mathrm{~m} / \mathrm{s}$, therefore this velocity does not apply to demyelinated fibres but to motor fibres. In addition to the phenomenon, that this second wave disappeared by high frequency stimulation and C6 ventral root lesioning, the potential of the second wave is considered to be trans-synaptic and to pass through motor fibres along the ventral root. Therefore, this wave potential represents motoneuron firing and is suitable to detect grey matter function.

There are some experimental reports about this potential. Owen ${ }^{3}$ and $\mathrm{Kai}^{4}$ reported that neurogenic motor evoked potentials (NMEP), recorded by responses over the sciatic nerve elicited by cord stimulation, are more sensitive to cord compression, ischaemia, and distraction, and more correlated with clinical motor function rather than spinal cord evoked potentials or somatosensory evoked potentials (SEP). Fukui evaluated tibial nerve potentials (TNP) evoked by stimulating the upper thoracic cord, and concluded that TNP mainly conducts antidromically in the dorsal column, partially involved potentials conducted from the ventral roots. ${ }^{5}$ Komori et al. recorded action potentials in the median nerve by stimulation to the upper cervical spinal cord which reflects the function of $\mathrm{C} 8$ grey matter, mainly the anterior horn. They named them descending segmental spinal cord evoked potentials (DS-SCEP). Under acute spinal compression and release at $\mathrm{C} 5 / 6$ level they reported that DS-SCEP were more sensitive than conductive spinal cord evoked potentials. ${ }^{6}$ Katsuta et $a l^{7}$ recorded evoked field potentials in the motoneuron pool at the C8 segment, and reported that these potentials decreased remarkably by compression of the C3 segment. These experiments were not aimed at the grey matter function influenced by compression of the specific segment where the motoneuron pool exists. The grey matter function under direct compression is not clear.

We noticed in the anatomical findings that motoneurons of the biceps brachii muscle branch of the musculocutaneous nerve mainly exist in the C6 segment of the spinal cord in cats. ${ }^{1,2} \mathrm{We}$ performed electrophysiological experimentals in which segmental cord compression affects the grey matter and white matter, using animal models. In our study, the second wave of Sp-PnEP did not disappear after dorsal lesioning and decreased by high-frequency stimulation, which proved that this wave was trans-synaptic motor tract pathway potential. The amplitude of this wave was significantly decreased by compression, compared to that of the first wave of Sp-SCEP. One reason for this phenomenon is considered, as follows: at first the grey matter had an abundant blood flow.
Sandler reported that grey matter had six times as much blood flow than white matter in primates, ${ }^{8}$ and Nakamura ${ }^{9}$ reported 10 times as posterior funiculus, and twenty times as anterior or lateral funiculus in cats. They both reported that the spinal cord blood flow significantly decreased after acute compression. Generally, grey matter is rather vulnerable to malcirculation of blood flow by compression and pathological degeneration, such as haemorrhagic necrosis, etc, occurs easily. It is notable that the grey matter showed an electrophysiologically sharp response to blood circulatory change under slight compression, which reveals no abnormal histological findings in our experiment. Secondly, Sp-PnEP is the pathway via synapse at the anterior horn of the grey matter, so the effectiveness of the signal conductivity is less than that of Sp-SCEP, axonal pathway.

Despite many experimental reports about spinal cord compression, the relative contribution of mechanical and vascular factors remains undefined.

Especially regarding mechanical factors, little has been discovered. Ichihara et $a l^{10}$ reported unique findings, that grey matter was stiffer and weaker than white matter, by their mechanical experiment using bovine spinal cord. We put a compression device, whose square surface was $5 \times 5 \mathrm{~mm}$, on the C6 segment centrally. The size of the C6 segment was approximately $8 \mathrm{~mm}$ transversely and $7 \mathrm{~mm}$ longitudinally. In axial section, the main part of the posterolateral funiculus and whole part of the anterior horn were compressed by the device. Although, we speculate almost the same force was added to both parts, the difference of responsibility to the shear force by the mechanical property of white matter and grey matter could not be proven in our experiment.

On the contrary, some findings about vascular factor were already reported. The blood supply decreased equally under acute compression of the white matter and the grey matter. ${ }^{9}$ While spinal cord axonal conduction, such as somatosensory evoked potentials, is resistant to failure within $20-30 \%$ of baseline blood flow, ${ }^{11}$ trans-synaptic potentials, such as motor evoked potentials, are more sensitive to cord ischaemia. $^{3,4}$ Since the second wave of Sp-PnEP is trans-synaptic, it is less resistant than the axonal potentials under the same ischaemic condition. The degree of the contribution of both factors is unclear.

In conclusion, our study shows that Sp-PnEP is more sensitive to acute compression on its own segment than Sp-SCEP. These results suggest that grey matter is more vulnerable to compression than white matter, which equally suggest synapses are more vulnerable than axons.

\section{References}

1 Thomas RC, Wilson VJ. Recurrent interactions between motoneurons of known location in cervical cord of the cat. $J$ Neurophysiol 1967; 30: $661-674$. 
2 Sterling P, Kuypers HGJM. Anatomical organization of the brachial spinal cord of the cat. II. The motoneuron plexus. Brain Research 1967; 4: 16-32.

3 Owen JH et al. Sensitivity and specificity of somatosensory and neurogenic motor evoked potentials in animals and humans. Spine 1988; 13: $1111-1116$

4 Kai Y et al. Relationship between evoked potentials and clinical status in spinal cord ischaemia. Spine 1995; 20: $291-296$.

5 Fukui Y. An experimental and clinical study of peripheral nerve potentials evoked by stimulating the spinal cord. Nippon Seikeigekagakkai Zasshi 1993; 67: 427-439.

6 Komori $\mathrm{H}$ et al. Comparison between descending segmental evoked spinal cord potentials and conductive spinal cord potentials under acute spinal cord compression. In: Jones SJ, Boyd S, Hetreed M, Smith NJ (eds). Handbook of Spinal Cord Monitoring. Kluwer Academic Publishers: Dordrecht, London 1994, pp 419-425.
7 Katsuta $\mathrm{M}$ et al. Effect of spinal compression on synaptic field potentials in the forelimb motoneuron pools evoked by stimulation of the descending axons of the propriospinal neurons. Nippon Seikeigekagakkai Zasshi 1998; 72: S1523.

8 Sandler AN, Tator CH. Regional spinal blood flow in primates. $J$ Neurosurg 1976; 45: 647-659.

9 Nakammura K et al. Experimental study of cervical spondylotic myelopathy. Spinal cord blood flow in cervical canal stenosis. Nippon Seikeigekagakkai Zasshi 1981; 55: 747-754.

10 Ichihara $\mathrm{K}$ et al. Mechanical property of the spinal cord. Rinshoseikeigeka 1999; 34: 549-551.

11 Carlson GD et al. Viscoelastic relaxation and regional blood flow response to spinal cord compression and decompression. Spine 1997; 22: $1285-1291$. 